\title{
Characteristics and outcomes of patients with multiple myeloma : Data from a developing country
}

\author{
Abdul Hameed*1 ${ }^{*}{\text { Jamshed } \text { Ali }^{1} \text {, Kiran Munawar }}^{1}$, Farah Arshad $^{1}$, Farhana Badar ${ }^{2}$, Neelam Siddiqui ${ }^{1}$
}

Received: 9 Jun 2017

Published: 1 Feb 2018

\section{Abstract}

Background: Multiple myeloma (MM) is a plasma cell disorder characterized by presence of monoclonal protein in serum or urine or both, increased bone marrow plasma cells, osteolytic lesion, hypercalcemia, and anemia. Several combination regimens are commonly recommended for treatment of multiple myeloma. The present study aimed at determining the characteristics and outcomes of patients with multiple myeloma treated at our centre.

Methods: During July 2012 and December 2015, all patients with proven diagnosis of MM were included in this study. Data were collected from hospital information system. The characteristics and outcomes of all patients were analyzed. Progression- free survival and overall survival of patients were also estimated. Kaplan-Meier curves and Log-rank test were applied and SPSS Version19 was used for data analysis.

Results: A total of 82 patients, with the median age of 51 years (Range: 23-64 yrs.) were available for final analysis. The number of patients with IgG and IgA type was $48(58.5 \%)$ and 15(18.3\%), respectively. There were $7(8.5 \%)$ patients with non-secretory type. Most of the patients ( $\mathrm{n}=59 ; 71.9 \%)$ were treated with CTD regimen and $13(15.8 \%)$ received bortezomib-based treatment. The median progression-free survival time was 30 months, and overall survival time was 48 months. The cumulative probability of survival at 36 months was $85 \%$.

Conclusion: Based on our results, the onset of multiple myeloma occurs in relatively younger age groups. A small number of patients received bortezomib due to cost issues. PFS and OS in our study were comparable with published literature.

Keywords: Multiple myeloma, Survival, Anemia, Bone disease, Plasma cells

Copyright $\odot$ Iran University of Medical Sciences

Cite this article as: Hameed A, Ali J, Munawar K, Arshad F, Badar F, Siddiqui N. Characteristics and outcomes of patients with multiple myeloma : Data from a developing country. Med J Islam Repub Iran. 2018 (1 Feb);32:1. https://doi.org/10.14196/mjiri.32.1

\section{Introduction}

Symptomatic multiple myeloma (MM) is a plasma cell disorder characterized by increased clonal plasma cells in bone marrow, which secrete monoclonal protein, and the presence of end organ damage including osteolytic lesion, anemia, hypercalcemia, and renal impairment (1). Males are predominantly affected by a median age of 70 years in Western countries, however, in some parts of the world, onset is seen in much younger age groups $(2,3)$. Multiple myeloma is the second most common hematological cancer after non-Hodgkin lymphoma (4). It accounts for approximately $1.8 \%$ of all new malignancies and $10 \%$ of all hematological cancers (4). Multiple myeloma is considered a treatable but incurable disease, and thus lifelong observation and follow- up are recommended (5). International staging system is widely used, which has recently

Corresponding author: Dr Abdul Hameed, hamiid34@gmail.com

1. Department of Medical Oncology, Shaukat Khanum Cancer Hospital and Research Centre, Pakistan.

2. Deapartment of Cancer Registry and Data Management, Shaukat Khanum Cancer Hospital and Research Centre, Lahore, Pakistan. been revised (6). Skeletal survey was a standard imaging in the past, but nowadays whole body MRI, low dose CT, and PET/CT are also being applied in the initial workup of patients with MM (7). On plain X-rays, bony lesions can only be seen when $20 \%$ to $30 \%$ bone is lost. However, with newer imaging techniques, even abnormal bone marrow infiltration can be picked up without any bone loss.

Initial workup should include complete blood count, routine biochemistry, $\mathrm{LDH}$, serum calcium, serum and urine electrophoresis, serum- free light chains, bone marrow aspirte/trephine, and cytogenetics. In addition, baseline imaging must be done according to availability (skeletal survey and whole body MRI). Anemia, high calcium, renal impairment, and bony lesions are included in end organ damage. The presence of any of these compli-

$\uparrow$ What is "already known" in this topic:

Outcomes of patients with multiple myeloma have improved significantly. CTD and VTD are standard first line therapies.

\section{$\rightarrow$ What this article adds:}

The onset of disease was observed in younger age groups in Pakistan. CTD is a cost- effective regimen with excellent results and is particularly useful in under- resourced countries. 
cations is an indication to start treatment. In addition, $>60 \%$ bone marrow plasma cells and serum- free light chains ratio of $\geq 100$, or presence of $>1$ focal lesion on MRI are also added in treatment indications (myeloma related events). The choice of treatment depends on age, stage of disease, stem cell transplant eligibility, performance status, and affordability of the patient (8). During the past 2 decades, several new therapeutic options including high dose therapy and autologous stem cells transplant (HDT/ASCT) and novel agents, such as immunemodulatory drugs (thalidomide, lenalidomide, and pomalidomide), and proteasome inhibitors (bortezomib, carfilzomib) have been introduced for the treatment of MM (9). Addition of these drugs has significantly improved the outcome of MM patients (10). MM patients are initially treated with primary induction therapy followed by HDT/ASCT in eligible patients and then by maintenance therapy in intermediate and high risk groups (11). There are several combinations available such as bortezomib, thalidomide plus dexamethasone (VTD), cyclophosphamide plus thalidomide and dexamethasone (CTD), lenalidomide, bortezomib, dexamethasone (RVD), thalidomide and dexamethasone (TD), melphalan combined with prednisone (MP), and melphalan-prednisone-thalidomide (MPT) (12). However, a 3-drug regimen is preferred. Bortezomib- based regimens are the first choice for patients with renal impairment (13). Several recommendations of MM diagnosis and treatment cannot be applied in low and middle income countries due to cost issues and availability of novel agents (8).

The present study aimed at determining the characteristics and outcomes of multiple myeloma patients treated in our center. We analyzed demographic features, progression- free survival, and overall survival of patients with MM treated with different available chemotherapeutic regimens.

\section{Methods}

This study was done in tertiary level cancer centre at Shaukat Khanum Memorial Cancer Hospital and Research Centre, Lahore, Pakistan. A total of 104 patients were registered with a proven diagnosis of multiple myeloma (MM)from July 2012 to Dec 2015.

Inclusion criteria were histopathological diagnosis of $\mathrm{MM}$, and receipt of chemotherapy in our hospital. We excluded patients who were lost to follow- up before treatment completion, as their survival intervals were not available; we also excluded patients with isolated plasmacytoma or asymptomatic myeloma (smouldering myeloma). Based on these criteria, 22 patients were excluded and 82 were included in the final analysis. This study was approved by the Institutional Review Board of the Shaukat Khanum Memorial Cancer Hospital and Research Centre, Lahore, Pakistan.

Diagnosis of multiple myeloma was made according to standard guidelines (14). Patient characteristics such as age, gender, Ig types (immunoglobuline), hemoglobin level at presentation, bony lytic lesions, and renal impairment were noted. Details regarding types of chemotherapy regimens were also recorded. Patients were divided into 3 groups according to chemotherapy regimens received. CTD (cyclophosphamide, thalidomide, dexamethasone), VTD (bortezomib, thalidomide, dexamethasone), and others (thalidomide and dexamethasone, or malphalan and prednisolon, or malphalan, prednisolon, and thalidomide). The criteria of International Myeloma Working Group (IMWG) were used to define response rate (15). Progression- free survival (PFS) was defined as the duration from start of the treatment to disease progression or death, whichever occured first. Overall survival was defined as the time interval from enrolment of the patient in the hospital to death from any cause or to last clinical follow- up.

\section{Statistical analysis}

Clinical data of the patients were extracted using a structured proforma and were then coded and entered into Microsoft excel. PFS interval was calculated using Kaplan-Meier survival analysis, and relapse was the end point of interest. We applied the Log-rank test and considered the results to be significant at an alpha level of 0.05 . SPSS Version 19 was used for data analysis.

\section{Results}

A total of 82 patients, with the mean age 51 years (Range 23-64 yrs.), were available for final analysis. Out of the 82 patients, 11 received autologous stem cell transplant. Subset analysis was not done for these transplant patients. Characteristics of patients are summarized in Table 1.

The median progression-free survival was achieved, and duration was 30 months. The endpoint of interest in PFS was disease relapse or progression. A total of 25 patients were found to have relapsed; $16(72.9 \%)$ patients in CTD cohort relapsed during the follow-up compared to 8 (38.5\%) in VTD (Table 2). The Log-rank (Mantel-Cox) was used to demonstrate the difference in survival distributions by treatment. Although more patients relapsed in

\begin{tabular}{lccc} 
Table 1. Patients and disease characteristics & & \\
\hline Variable & Level & Number & Percent \\
\hline Gender & Male & 46 & 56.1 \\
Myeloma type & Female & 36 & 43.9 \\
& IgG type & 48 & 58.5 \\
& IgA type & 15 & 18.4 \\
& Light Chain & 12 & 14.6 \\
ISS staging & Non Secretory & 7 & 8.5 \\
& Stage I & 21 & 25.7 \\
\multirow{4}{*}{ Hemoglobin level } & Stage II & 28 & 34.1 \\
& Stage III & 33 & 40.2 \\
Myeloma bone disease & $<10$ & 36 & 43.9 \\
& $\geq 10$ & 46 & 56.1 \\
Renal impairment & Yes & 65 & 79.2 \\
& No & 19 & 20.8 \\
Treatment type & Yes & 30 & 36.6 \\
& No & 52 & 63.4 \\
& CTD & 59 & 71.9 \\
Stem cells transplant & VTD & 13 & 15.8 \\
\hline
\end{tabular}




\begin{tabular}{lcccc} 
Table 2. PFS by therapy & & & \\
\hline Therapy & Total N & N of Events & \multicolumn{2}{c}{ Censored } \\
\cline { 3 - 5 } & & & $\mathrm{N}$ & Percent \\
CTD & 59 & 16 & 43 & 72.9 \\
VTD & 13 & 8 & 5 & 38.5 \\
Other & 10 & 1 & 9 & 90.0 \\
Overall & 82 & 25 & 57 & 69.5 \\
\hline
\end{tabular}

Table 3. Overall survival by therapy

\begin{tabular}{lcccc}
\hline Therapy & Total N & N of Events & \multicolumn{2}{c}{ Censored } \\
\cline { 3 - 5 } CTD & 59 & 8 & $\mathrm{~N}$ & Percent \\
VTD & 13 & 1 & 51 & 86.4 \\
Other & 10 & 1 & 12 & 92.3 \\
Overall & 82 & 10 & 72 & 90.0 \\
\hline
\end{tabular}

CTD group compared to VTD group, this difference was not statistically significant $(\mathrm{p}=0.886)$ at an alpha-level of 0.05, as presented in Kaplan- Meier graph (Fig. 1).

The overall survival time was 48 months. A total of 10 patients died in this study (Table 3). The cumulative probability of survival rate at 36 months was $85 \%$. The Logrank test (Mantel-Cox) was used to demonstrate the difference in survival distributions by treatment. There was clinical difference in OS of these groups; however, no statistically significant difference was found in OS by therapy $(\mathrm{p}=0.795)$, as demonstrated in the Kaplan- Meier

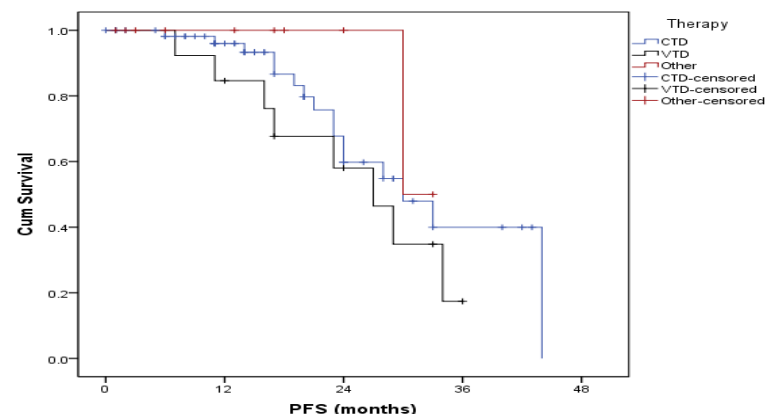

Fig. 1. Progression-free survival of multiple myeloma patients treated with different chemotherapy regimens

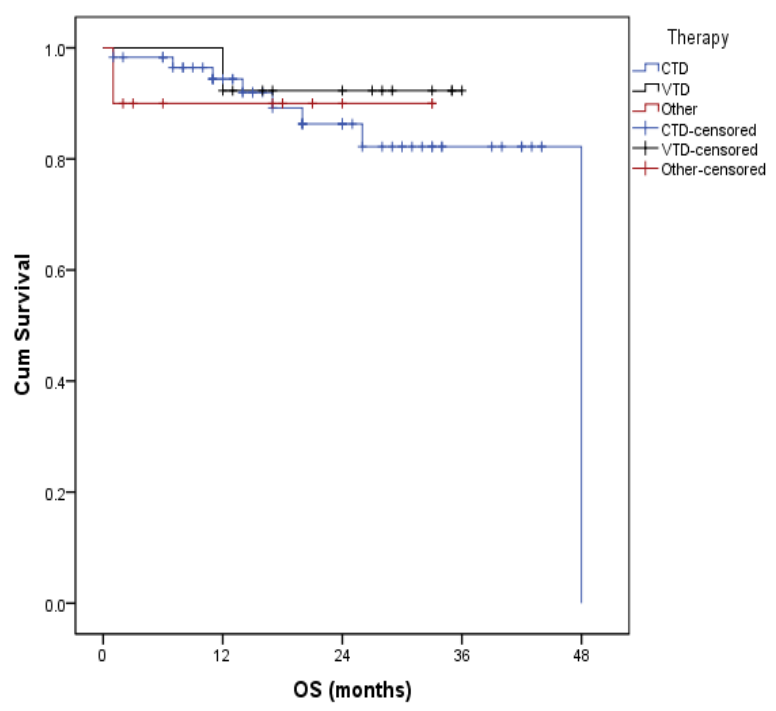

Fig. 2. Overall survival of multiple myeloma patients treated with different chemotherapy regimens graphs (Fig. 2).

\section{Discussion}

Multiple myeloma (MM) is a plasma cell disorder characterized by presence of monoclonal protein in serum or urine or both with increased bone marrow clonal plasma cells, and it is associated with end organ damage (1). Although this is a disease of the elderly, it is also seen in younger age groups. Younger patients have higher survival rate as compared to the elderly (16). Spectrum of disease ranges from asymptomatic monoclonal gammopathy of unknown significance to highly fatal condition of plasma cell leukemia (17).

International Myeloma Working Group recently updated indications to start treatment in MM patients. Some laboratory and radiographic variables are added to existing CRAB features (11). These features include bone marrow plasma cells $\geq 60 \%$, abnormal MRI with more than one focal lesion, with each lesion $>5 \mathrm{~mm}$, and involved/uninvolved serum free light chain ratio $\geq 100$.

The therapy of individual patient is based on several factors including, age, symptoms, laboratory parameters, cytogenetic, goals of treatment, previous myeloma treatment, quality of life, and personal preferences. Paradigm of MM management evolved by introducing novel drugs including proteasome inhibitors and immunomodulatory drugs. These agents have increased the response rates before and after autologous hematopoietic stem cell transplantation (ASCT) (18).

Bortezomib, thalidomide, or lenalidomide- based regimens followed by ASCT are standard approaches for transplant eligible patients. Combination therapy with melphalan and prednisone with either thalidomide, lenalidomide, or bortezomib is recommended in transplant ineligible patients (19). Maintenance therapy with lenalidomide has shown promising results in different studies (20) .

The goal of front line therapy for multiple myeloma is to decrease tumor load either in preparation for autologous stem cells transplantation with high- dose melphalan therapy or to provide long- term disease control with reduction in disease burden, which is associated with improved outcome including prolonged progression- free survival and overall survival in either ASCT candidate or otherwise (21).

In our part of the world, many recommendations of di- 
agnosis and treatment of multiple myeloma are difficult to be applied due to limited resources and approaches to novel agents (8).

In a previous study, PFS with CTD in patients who did not receive HDT/ASCT was 27 months and OS was 50 months. In the same study, in the subset of patients who received ASCT after induction chemotherapy with CTD, PFS was 33 months (22) Rosinol et al. (23) and Cavo et al. (24) suggested better outcomes with bortezomib- based regimen compared to other combinations.

Our study showed that the median age at the onset of the disease was 51 years, which is much younger compared to the Western data. Similar age of onset has been noticed in our part of the world in previous publications $(2,3)$.

Prevalence of MM was more in males as expected (25). IgG paraprotein was the commonest type, which is also consistent with the established data (26). The majority of the patients were in the advanced stage of the disease (ISS, II/III). One reason could be that due to lack of wellorganised community services, most patients are diagnosed very late during the disease course. In this study, nearly $80 \%$ of patients suffered from myeloma bone disease, and this correlates with published evidence. This number may go up if whole body MRI or PET/CT are used at initial workup (7), which is not common in our hospital at this point. Due to unavailability of whole body MRI in many centres, many patients will be undertreated.

Due to cost issues, bortezomib was used only in few patients and more than $70 \%$ of patiens were treated with CTD regimen. PFS and OS in our study are comparable with published data for CTD (22). There was no statistically significant difference in PFS or OS in different treatment groups. One possible explanation could be small number of patients in other groups compared to CTD. Although our group consisted of mostly transplant eligible patients, only 11 patients underwent HDT/ASCT. There were different reasons for not doing upfront transplant including the cost of the procedure for those patients who were paying for their treatment. In our centre, patients come from far areas and after receiving induction therapy, they prefer to go back home rather than stay further for transplant; moreover, some patients refuse treatment because of complications that may occur during HDT/ASCT.

\section{Limitations of the study}

There were some limitations in this study. This was a retrospective analysis, and most of the patients were treated with CTD. Moreover, subgroup analysis of transplanted patients was not done, and there was no information on complications of each treatment group. Moreover, we did not look at the effects of different factors on PFS and OS (Our group has published a paper about the impact of different factors on the outcomes of MM patients (27).

\section{Conclusion}

Based on our results, onset of multiple myeloma occurs in a relatively younger age group and the majority present with advanced stage. Bortezomib is not readily available to all patients due to cost issues, and thus CTD remains an effective therapy in treating native patients.

There is a need to take serious measures in under- resourced countries to improve care provided to MM patients. With new diagnostic criteria, lack of whole body MRI and serum- free light chain assay facilities in many centers will result in undertreatment of many patients, leading to poor outcomes. Cost and availability of novel agents in the developing world are also major obstacles in providing high quality care to patients with multiple myeloma.

\section{Acknowledgements}

We are grateful to Mr. Abu Ul Hassan at Department of Medical Oncology, Shaukat Khanum Memorial Cancer Hospital \& Research Centre for his input in preparation of this manuscript.

\section{Conflict of Interests}

The authors declare that they have no competing interests.

\section{References}

1. Bataille R, Harousseau JL. Multiple Myeloma. New Engl J Med. 1997 Jun 5;336(23):1657-64.

2. Sultan S, Irfan SM, Parveen S, Ali H, Basharat M. Multiple Myeloma: A retrospective analysis of 61 patients from a tertiary care center. Asian Pacific J Cancer Prevent. 2016 Jun 1;17(4):1833-5.

3. Lu J, Lu J, Chen W, Huo Y, Huang X, Hou J. Clinical features and treatment outcome in newly diagnosed Chinese patients with multiple myeloma: Results of a multicenter analysis. Blood Cancer J. 2014 Aug 15;4(8):e239.

4. Dimopoulos MA, Terpos E. Multiple myeloma. Annal Oncol. 2010 Oct 1;21(Supplement 7):vii143-50.

5. National Comprehensive Cancer Network (NCCN). Clinical practice guidelines in oncology (NCCN guidelines): multiple myeloma. V.2.2016. Available at:http://www.nccn.org/professionals/physician gls/PDF/myeloma.pdf.

6. Palumbo A, Avet-Loiseau H, Oliva S, Lokhorst HM, Goldschmidt H, Rosinol L, et al. Revised international staging system for multiple Myeloma: A report from international Myeloma working group. J Clin Oncol. 2015 Aug 3;33(26):2863-9.

7. Terpos E, Dimopoulos MA, Moulopoulos LA. The Role of Imaging in the Treatment of Patients With Multiple Myeloma in 2016. Am Soc Clin Oncol Educ Book. 2016;35:e407-17.

8. Tan D, Chng WJ, Chou T, Nawarawong W, Hwang SY, Chim CS, et al. Management of multiple myeloma in Asia: Resource-stratified guidelines. The Lancet Oncology. 2013 Nov;14(12):e571-81.

9. Lonial S, Boise LH, Kaufman J. How I treat high-risk myeloma. Blood. 2015 Aug 13;126(13):1536-43.

10. Sonneveld P, Broijl A. Treatment of relapsed and refractory multiple myeloma. Haematologica. 2016 Mar 31;101(4):396-406.

11. Rajkumar SV. Multiple myeloma: 2014 update on diagnosis, riskstratification, and management. Am J Hematol. 2014 Sep 16;89(10):998-1009

12. Ludwig H, Beksac M, Blade J, Boccadoro M, Cavenagh J, Cavo M, et al. Current multiple Myeloma treatment strategies with novel agents: A European perspective. The Oncologist. 2010 Jan 1;15(1):625.

13. Zhu W, Chen W. Bortezomib-based treatment for multiple myeloma patients with renal impairment. Medicine. 2016 Nov;95(46):e5202.

14. Moreau P, San Miguel J, Ludwig H, Schouten H, Mohty M, Dimopoulos M, et al. Multiple myeloma: ESMO clinical practice guidelines for diagnosis, treatment and follow-up. Annals Oncol. 2013 Aug 16;24(suppl 6):vi133-7.

15. Kumar S, Paiva B, Anderson KC, Durie B, Landgren O, Moreau P, et al. International Myeloma working group consensus criteria for response and minimal residual disease assessment in multiple myeloma. The Lancet Oncology. 2016 Aug;17(8):e328-46. 
16. Available from: http://seer.cancer.gov/statfacts/html/mulmy.html.

17. Agarwal MB. Multiple Myeloma: Treatment is getting individualized. Indian J Hematol Blood Transfus. 2015 Jul 26;32(1):3-9.

18. Larsen JT, Kumar S. Evolving paradigms in the management of multiple Myeloma: Novel agents and targeted therapies. Rare Cancer Ther. 2015 Aug 28;3(1-2):47-68.

19. Koeppen S. Treatment of multiple Myeloma: Thalidomide-, Bortezomib-, and Lenalidomide-Induced peripheral neuropathy. Oncol Res Treat. 2014 Aug 14;37(9):506-13.

20. Lipe B, Vukas R, Mikhael J. The role of maintenance therapy in multiple myeloma. Blood Cancer J. 2016 Oct 21;6(10):e485.

21. Palumbo A, Anderson K. Multiple Myeloma. New Engl J Med. 2011 Mar 17;364(11):1046-60.

22. Morgan GJ, Davies FE, Gregory WM, Bell SE, SzubertAJ, Navarro Coy N, et al. Cyclophosphamide, thalidomide, and dexamethasone as induction therapy for newly diagnosed multiple myeloma patients destined for autologous stem-cell transplantation: MRC Myeloma IX randomized trial results. Haematologica. 2011 Nov 4;97(3):442-50.

23. Rosinol L, Oriol A, Teruel AI, Hernandez D, Lopez-Jimenez J, de la Rubia J, et al. Superiority of bortezomib, thalidomide, and dexamethasone (VTD) as induction pretransplantation therapy in multiple myeloma: A randomized phase 3 PETHEMA/GEM study. Blood. 2012 Jul 12;120(8):1589-96.

24. Cavo M, Tacchetti P, Patriarca F, Petrucci MT, Pantani L, Galli M, et al. Bortezomib with thalidomide plus dexamethasone compared with thalidomide plus dexamethasone as induction therapy before, and consolidation therapy after, double autologous stem-cell transplantation in newly diagnosed multiple myeloma: A randomised phase 3 study. The Lancet. 2010 Dec;376(9758):2075-85.

25. Becker N. Epidemiology of multiple myeloma. Recent Results Cancer Res. 2011;183:25-35. doi: 10.1007/978-3-540-85772-3 2.

26. Lu J, Lu J, Chen W, Huo Y, Huang X, Hou J. Clinical features and treatment outcome in newly diagnosed Chinese patients with multiple myeloma: Results of a multicenter analysis. Blood Cancer J. 2014 Aug 15;4(8):e239.

27. Basit A, Siddiqui N, Hameed A, Muzaffar N, Athar S. Factors affecting outcome of patients with multiple myeloma. J Ayub Med Coll Abbottabad 2014;26(3):376-9. 